\title{
Morphological Changes in a Pyogenic Granuloma of the Esophagus Observed over Three Years
}

\author{
Masaya Iwamuro ${ }^{1}$, Hiroyuki Okada ${ }^{2}$, Takehiro Tanaka ${ }^{3}$, Keisuke Hori ${ }^{4}$, Masahide Kita ${ }^{4}$, \\ Seiji Kawano ${ }^{2}$, Yoshiro Kawahara ${ }^{2}$ and Kazuhide Yamamoto $^{4}$
}

\begin{abstract}
A 78-year-old Japanese man underwent routine esophagogastroduodenoscopy, during which a reddish, flat elevated lesion was observed in the middle third of the esophagus. A bright red area of protrusion appeared in the center of the elevated lesion three months later, and the protruded nodule grew to be a blood blister six months after the initial endoscopy examination. The morphology of the lesion changed from a protruded nodule to a mass with two humps that subsequently returned to a single hump during the three-year observation period. A histological diagnosis of pyogenic granuloma was made based on a biopsy performed at six months. This report illustrates a rare case of an esophageal pyogenic granuloma presenting with a unique endoscopic appearance and morphologic changes. Endoscopic ultrasonography images are also presented.
\end{abstract}

Key words: pyogenic granuloma, lobular capillary hemangioma, gastrointestinal neoplasms, esophageal neoplasms

(Intern Med 54: 1737-1740, 2015)

(DOI: 10.2169/internalmedicine.54.4265)

\section{Introduction}

Pyogenic granuloma is a benign tumor categorized as a hemangioma. The lesion usually occurs on the skin as well as the mucosal surface of the oral cavity (1-3). The occurrence of pyogenic granuloma in the gastrointestinal tract is infrequent, with only approximately 50 cases reported to date (1). Among sites associated with the alimentary tract, the esophagus is involved in approximately half of reported cases. Due to the low prevalence of the disease, the natural course of esophageal pyogenic granuloma has not been fully clarified.

We recently experienced a patient with pyogenic granuloma in the esophagus who presented with a reddish, flat elevated lesion in the esophagus. Interestingly, a bright red protrusion emerged three months later and the protruded nodule subsequently became enlarged at six months, at which time a diagnosis of esophageal pyogenic granuloma was made histologically. Although histopathological evalu- ations using biopsy specimens are required to obtain a definitive diagnosis, rapid growth and a metamorphic nature can be diagnostic clues to this disease. In this paper, we present the morphologic changes of an esophageal pyogenic granuloma observed over three years.

\section{Case Report}

A 78-year-old Japanese man underwent esophagogastroduodenoscopy at his family clinic for a routine health checkup. Endoscopy revealed multiple reddish areas without erosions or ulcers. He was therefore referred to our hospital for a further investigation of gastritis. The patient was currently healthy and had been taking no medications. A physical examination revealed no abnormalities, and there were no vascular lesions on the buccal mucosa or skin. All laboratory findings were within the normal ranges, except for positive Helicobacter pylori IgG antibodies. Esophagogastroduodenoscopy performed at our hospital showed reddish areas in the stomach, and a diagnosis of benign gastritis asso-

\footnotetext{
${ }^{1}$ Department of Molecular Hepatology, Okayama University Graduate School of Medicine, Dentistry, and Pharmaceutical Sciences, Japan, ${ }^{2}$ Department of Endoscopy, Okayama University Hospital, Japan, ${ }^{3}$ Department of Pathology, Okayama University Hospital, Japan and ${ }^{4}$ Department of Gastroenterology and Hepatology, Okayama University Graduate School of Medicine, Dentistry, and Pharmaceutical Sciences, Japan Received for publication October 12, 2014; Accepted for publication November 30, 2014

Correspondence to Dr. Masaya Iwamuro, iwamuromasaya@yahoo.co.jp
} 


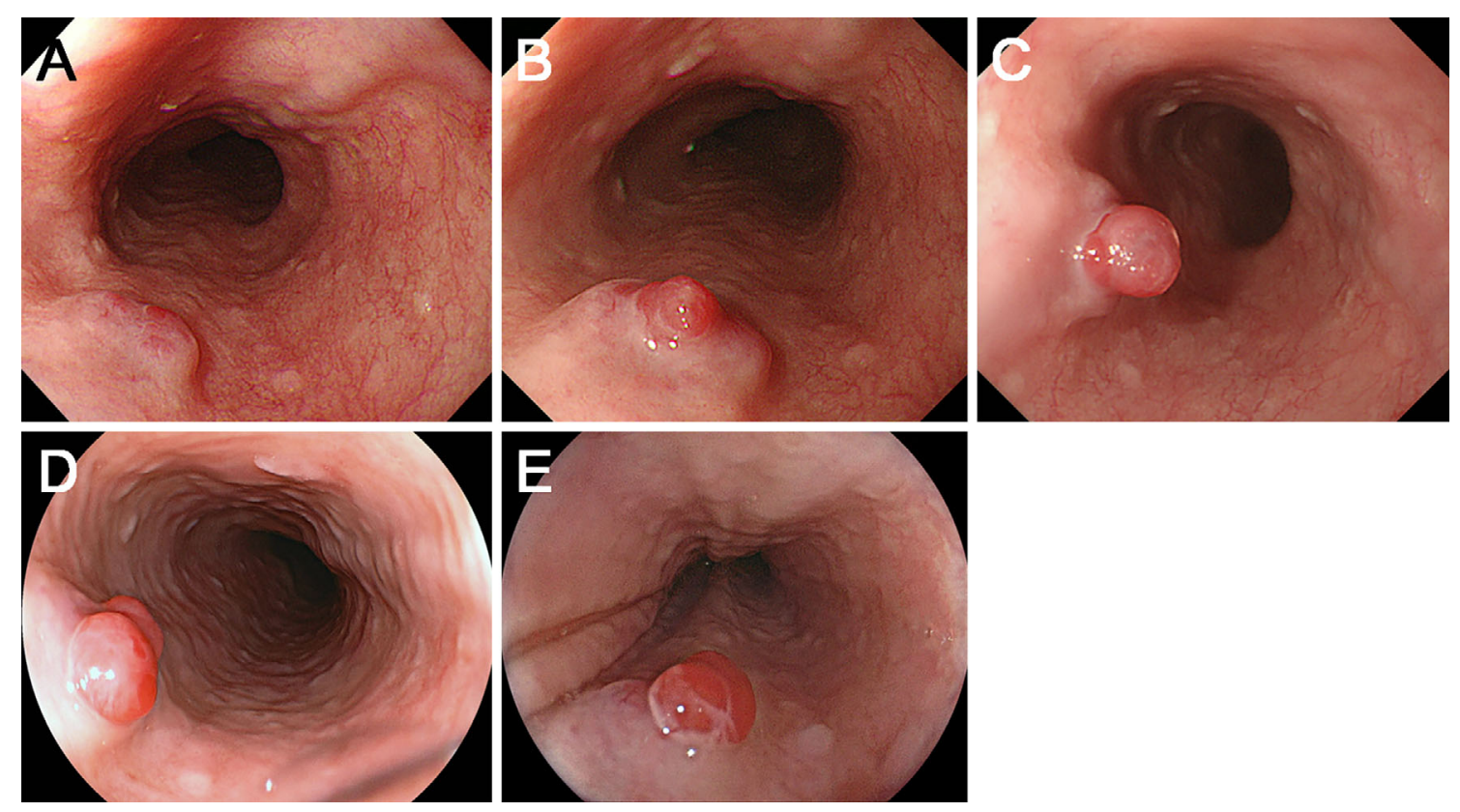

Figure 1. Esophagogastroduodenoscopy images. A reddish, flat elevated lesion measuring approximately $10 \mathrm{~mm}$ in diameter was seen in the middle third of the esophagus (A). Three months later, a bright red protrusion resembling a blood blister emerged at the center (B). The protruded nodule became enlarged six months after the initial endoscopy examination (C). The morphology of the lesion changed from a protruded nodule to two humps at 25 months (D) and a single hump at 39 months after the initial examination (E).
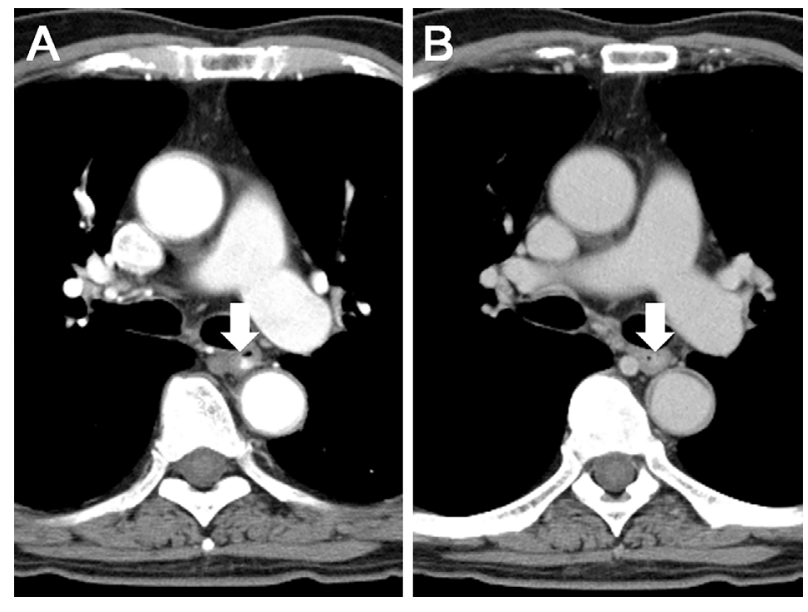

Figure 2. CT scanning images. Six months after the initial endoscopy examination, CT scanning showed strong enhancement of the esophageal nodule with contrast media in the arterial phase (A). The tumor was slightly enhanced in the equilibrium phase $(B)$.

ciated with Helicobacter pylori infection was made based on a biopsy of the gastric mucosa. An endoscopic examination also showed a reddish, flat elevated lesion measuring approximately $10 \mathrm{~mm}$ in diameter in the middle third of the esophagus (Fig. 1A). A biopsy of the esophageal lesion was not performed in order to prevent massive bleeding, as the lesion was suspected to be a vascular tumor due to its reddish surface. Colonoscopy showed no abnormalities, whereas enteroscopy was not performed. Three months later, a bright red protrusion resembling a blood blister appeared in the center of the elevated lesion (Fig. 1B). Esophagogastroduodenoscopy performed six months after the initial examination showed that the protruded nodule had become enlarged (Fig. 1C). CT scanning revealed the tumor to be strongly enhanced with contrast media in the arterial phase and slightly enhanced in the equilibrium phase (Fig. 2). The enhancement pattern was quite similar to that of normal arteries, suggesting that the lesion was a vascular tumor. Endoscopic ultrasonography visualized the esophageal mass as a homogenous, hyperechoic lesion primarily confined to the mucosal layer (Fig. 3). A biopsy of the lesion was performed for the first time six months after its discovery, which revealed a highly vascular proliferation with inflammatory cell infiltration in the deeper stroma and edema in the shallow stroma, whereas superficial epithelial cells remained intact (Fig. 4). Consequently, a diagnosis of an esophageal pyogenic granuloma was made.

It was determined that the patient should be managed by a strategy of watchful waiting, rather than prompt radical treatment, such as endoscopic submucosal dissection or mucosal resection, because he had no symptoms or history of gastrointestinal bleeding. The morphology of the esophageal lesion changed from a protruded nodule to two humps at 25 months (Fig. 1D) and returned to a single hump 39 months after the initial examination (Fig. 1E).

\section{Discussion}

Pyogenic granuloma is a polypoid form of vascular growth that mostly occurs on the skin and mucosal surface. 

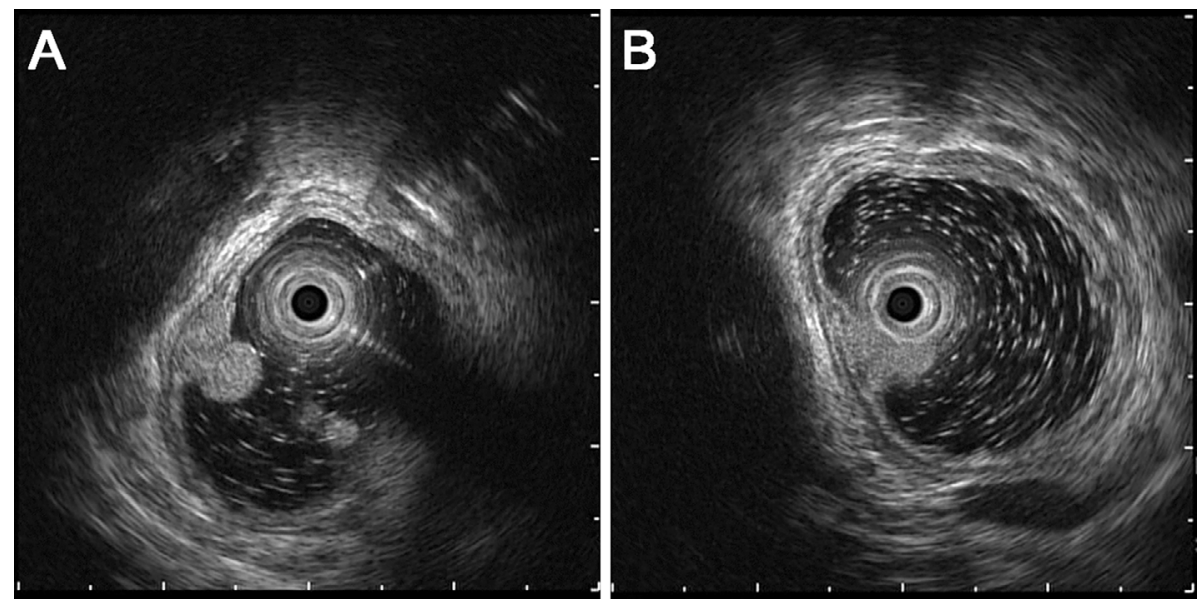

Figure 3. Endoscopic ultrasonography images. A homogenous, hyperechoic lesion was observed (A). The tumor was primarily confined to the mucosal layer (B).
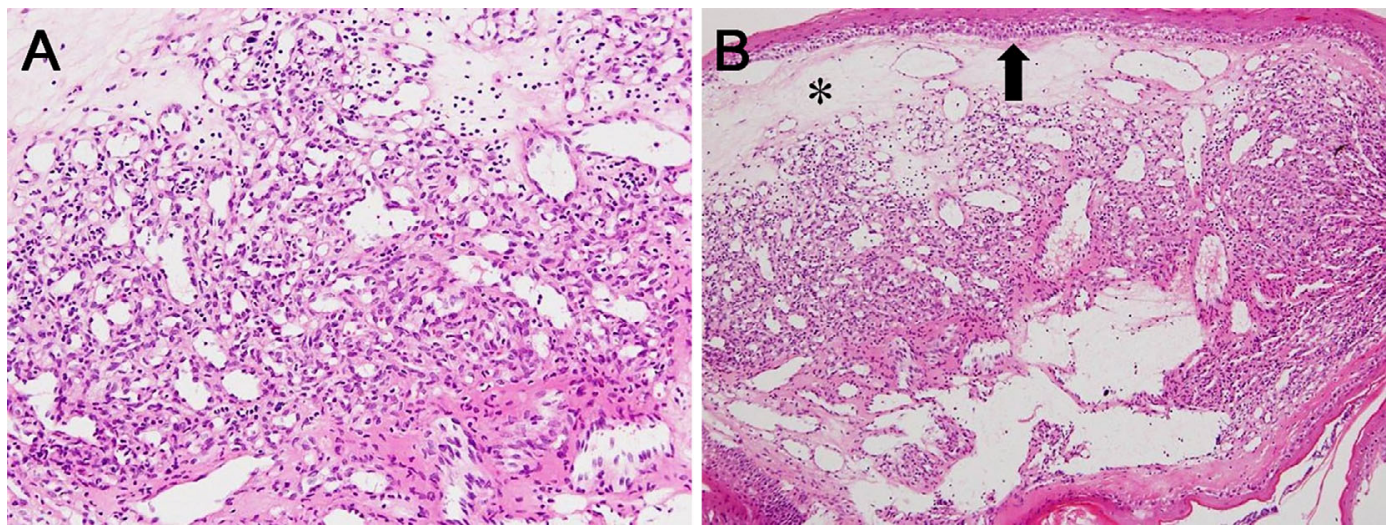

Figure 4. Histological images. A biopsy revealed highly vascular proliferation with inflammatory cell infiltration in the deeper stromal areas (A). Edema was also seen in the shallow stromal areas (B, asterisk). The superficial epithelial cells remained intact $(B$, arrow).

Pathologically, the lesion is characterized by highly vascular proliferation resembling that of granulation tissue $(2,3)$. Edematous stroma with chronic inflammatory cell infiltration and micro-abscess formation are often observed, as in the present case. However, in general, pus and infection with pyogenic organisms are not present in the lesion, and thus the term "pyogenic granuloma" does not precisely match the pathological features $(2,3)$. In this context, some pathologists prefer the term "granulation tissue-type hemangioma" for the pathological diagnosis of this disease entity.

In the present patient, the esophageal lesion showed changes in its morphology during the first six months after its discovery from a reddish, flat elevated lesion to a bright red protrusion (Fig. 1A-C). A similar case involving the short-term development of an esophageal pyogenic granuloma in a 63-year-old man was reported by Tajika et al. (4). It is believed that pyogenic granuloma formation occurs via a process of reactive hyperplasia as a result of traumatic injury, local irritation or hormonal influences $(2,5)$. Injuries and burns to the skin and/or mucosa may provoke tissue repair and the subsequent exuberant reactive proliferation of blood vessels and migration of fibroblasts, thus leading to the development of pyogenic granuloma. Pyogenic granulomas of the skin and mouth mucosa are found more frequently during pregnancy, thus indicating that hormonal effects may also play a role in its pathogenesis (6). Because the patient reported by Tajika et al. had severe esophagitis as a post-surgical complication of proximal gastrectomy, gastroesophageal reflux and chronic damage to the esophageal mucosa induced by gastric acid likely gave rise to the pyogenic granuloma observed in that case. Although reflux esophagitis was not detected in the current case and there was no identifiable cause of the lesion based on the findings of medical examinations, including endoscopy and an interview, diets with stimulative effects, such as spicy foods, hot drinks and alcohol, may damage the esophageal mucous membrane (7).

Macroscopically, esophageal pyogenic granulomas are observed as protruding tumors or submucosal tumor-like lesions (1). The surface of these lesions ranges from pale pink to dark red in color, and the masses are sometimes accompanied by whitish or yellowish exudates (8). The presence 
of such whitish or yellowish deposits on the mucosa is reportedly a diagnostic clue for pyogenic granuloma, with a sensitivity and specificity of both $100 \%(9,10)$, although the present patient lacked this feature. Rapid enlargement and a metamorphic nature are also characteristic features of pyogenic granulomas (10). Mechanical irritation, trauma, biopsies and hormonal factors, as described above, may affect the morphology of the lesion, resulting in a transformed appearance. Therefore, rapid growth and metamorphism are further diagnostic clues in cases lacking whitish or yellowish exudates, as in the present patient.

Seoung et al. reported two cases of esophageal pyogenic granulomas showing homogeneously hyperechoic internal density on endoscopic ultrasonography and speculated that hyperechoic areas reflect the presence of abundant blood vessels, as seen in cases of hemangiomas $(1,11)$. However, Rolanda et al. reported a case involving different ultrasonographic features of a hypoechoic mass (10). Consequently, the endoscopic ultrasonographic features of this disease remain debatable.

The differential diagnoses of pyogenic granuloma include hemangioma, angiosarcoma, conventional granulation tissue, inflammatory fibroid polyps, bacillary angiomatosis, Kaposi's sarcoma, metastatic cancer and lymphoma $(2,8,10)$. Although biopsies are essential for establishing the diagnosis, the sampling procedure must be performed carefully, as pyogenic granulomas are friable, highly vascular lesions and bleed easily. Once the diagnosis of pyogenic granuloma is made, the management strategy generally depends on the severity of the patient's symptoms. Clinical observation and follow-up are acceptable if the pyogenic granuloma is small and free of bleeding (2). Other treatment options include removing any etiological factors and performing surgical excision. It should be noted that incomplete excision, repeat injury of the area or the failure to remove causative irritants can result in local recurrence (12-14). Recurrent lesions sometimes emerge in deeper layers of the skin or mucosa (15). In cases of esophageal pyogenic granulomas, the tumor is often resected endoscopically or surgically to prevent gastrointestinal bleeding $(8,10)$. The present patient is currently under clinical observation, as he has reported no episodes of bleeding. Endoscopic snare polypectomy or submucosal dissection will be performed as a curative treatment if the lesion bleeds, since the mass is primarily confined to the mucosal layer, as demonstrated on ultrasonography (Fig. 2).

In conclusion, we observed a patient with an esophageal pyogenic granuloma over the course of three years since initial endoscopic detection. The endoscopic appearance and morphologic changes, indicating the emergence of a protruded nodule, are considered to be unique to this disease. Although obtaining a histological diagnosis of biopsy specimens is essential, the morphologic features described in this report are expected to help physicians diagnose this rare disease.

The authors state that they have no Conflict of Interest (COI).

\section{References}

1. Seoung HG, Kim GH, Song GA, et al. Esophageal pyogenic granuloma: endosonographic findings and endoscopic treatments. Clin Endosc 46: 81-84, 2013.

2. Jafarzadeh H, Sanatkhani M, Mohtasham N. Oral pyogenic granuloma: a review. J Oral Sci 48: 167-175, 2006.

3. Epivatianos A, Antoniades D, Zaraboukas T, et al. Pyogenic granuloma of the oral cavity: comparative study of its clinicopathological and immunohistochemical features. Pathol Int 55: 391397, 2005.

4. Tajika M, Nakamura T, Kawai H, et al. Short-term development of esophageal pyogenic granuloma observed on endoscopy. Gastrointest Endosc 64: 269-270, 2006.

5. van Eeden S, Offerhaus GJ, Morsink FH, van Rees BP, Busch OR, van Noesel CJ. Pyogenic granuloma: an unrecognized cause of gastrointestinal bleeding. Virchows Arch 444: 590-593, 2004.

6. Fernandez A, Hamilton J, Nach R. Two cases of pyogenic granuloma in pregnancy. Ear Nose Throat J 93: 302-303, 2014.

7. Kikuchi A, Sujino T, Yamaoka M, et al. Pyogenic granuloma of the ileum diagnosed by double-balloon enteroscopy. Intern Med 53: 2057-2059, 2014.

8. Kusakabe A, Kato H, Hayashi K, et al. Pyogenic granuloma of the stomach successfully treated by endoscopic resection after transarterial embolization of the feeding artery. J Gastroenterol 40: 530535, 2005.

9. Serban DE, Florescu P. Colonic pyogenic granuloma in children: a rare or rarely recognized entity. Am J Gastroenterol 98: 21062107, 2003.

10. Rolanda C, Gonçalves R, Macedo G. A metamorphic lesion. Gastroenterology 137: 41, 395, 2009.

11. Paltiel HJ, Burrows PE, Kozakewich HP, Zurakowski D, Mulliken JB. Soft-tissue vascular anomalies: utility of US for diagnosis. Radiology 214: 747-754, 2000.

12. Okada N, Matsumoto $T$, Kurahara $K$, et al. Pyogenic granuloma of the esophagus treated by endoscopic removal. Endoscopy 35: 375, 2003.

13. Bhaskar SN, Jacoway JR. Pyogenic granuloma: clinical features, incidence, histology, and result of treatment: report of 242 cases. J Oral Surg 24: 391-398, 1966.

14. Patrice SJ, Wiss K, Mulliken JB. Pyogenic granuloma (lobular capillary hemangioma): a clinicopathologic study of 178 cases. Pediatr Dermatol 8: 267-276, 1991.

15. Taira JW, Hill TL, Everett MA. Lobular capillary hemangioma (pyogenic granuloma) with satellitosis. J Am Acad Dermatol 27: 297-300, 1992.

(C) 2015 The Japanese Society of Internal Medicine http://www.naika.or.jp/imonline/index.html 